\title{
Une Remarque sur le Prolongement Analytique d'une Fonction Méromorphe
}

\author{
Par \\ Susumu IsonAGA*)
}

1. Sur une variété analytique complexe élémentaire d'une certaine sorte, toute fonction méromorphe dans un voisinage d'un ensemble analytique compact de dimension positive se prolonge analytiquement sur toute la variété. (Voir par example [1], [2], [4] et [5].) D'autre part, M. Takeuchi [6] et $\mathrm{M}^{\mathrm{me}}$ Fujita [3] ont indiqué que tout domaine pseudoconvexe sans point critique intérieur étalé au-dessus de l'espace projectif complexe $\boldsymbol{P}^{n}(n>1)$ est holomorphiquement complet pourvu que ce domaine ne coïncide pas avec $\boldsymbol{P}^{n}$. Cela signifie que l'énoncé ci-dessus est valable pour $\boldsymbol{P}^{n}$ puisqu'un domaine de méromorphie est pseudoconvexe et qu'un domaine holomorphiquement complet ne peut contient aucun ensemble analytique compact de dimension positive. Le but de la note actuelle est d'indiquer l'énoncé suivant. Soit $X$ un espace produit d'un nombre fini d'espaces projectifs complexes $\boldsymbol{P}^{m_{i}}$ de dimension $m_{i}$, où $\sum m_{i}>1$. Alors, tour domaine pseudoconvexe sans point critique intérieur $\mathscr{D}$ étalé au-dessus de $X$ ne contient aucun ensemble analytique compact $A$ tel que la projection de $A$ dans chaque $\boldsymbol{P}^{m_{\imath}}$ soit de dimension positive pourvu que $\mathscr{D}$ ne conïcide pas avec $X$. Cela signifie aussi que toute fonction méromorphe dans un voisinage de tel $A$ se prolonge analytiquement sur tout $X$.

2. En considérant $r$ espaces projectifs $\boldsymbol{P}^{m_{i}}(i=1, \ldots, r)$ de dimension $m_{i}$, soit $X$ l'espace produit de $\boldsymbol{P}^{m_{i}}(i=1,, r)$. On suppose que l'on ait $\sum m_{i}>1$. Pour chaque $i, p_{i}$ est la projection de $X$ sur $\boldsymbol{P}^{m_{i}}$

Communiqué par S. Nakano, le 4 décembre 1973.

*) L'auteur est mort en mars 1973. Ce mémoire a été rédigé d'après son cahier laissé par T. Nishino et T. Terada, Département de Mathématique, Université de Kyoto, Kyoto. 
qui fait correspondre à un point $a_{1} \times \cdots \times a_{r}\left(a_{j} \in \mathbb{P}^{m_{j}}\right)$ le point $a_{i}$ de $\mathbb{P}^{m_{i}}$. Soit $\mathscr{D}$ un domain sans point critique intéreur étalé au-dessus de $X$; c'est-à-dire $\mathscr{D}$ est une variété analytique complexe ayant une application analytique, dite projection et notée $\pi$, de $\mathscr{D}$ dans $X$, qui est localement biunivoque. Pour un point $a$ de $\mathscr{D}$, on désigne par $\underline{a}$ l'image de $a$ par $\pi$. On suppose que $\mathscr{D}$ est connexe.

On appelle disque analytique dans $\mathscr{D}$ l'image dans $\mathscr{D}$ du cercle unité $\mathfrak{c}:|z|<1$ sur le plan d'une variable complexe $z$ par une application $\varphi$ holomorphe dans $c$ ef continue en $\bar{r}:|z| \leqq 1$. Lorsque $\varphi$ est donné par des fonctions linéaires de $z$ pour un système de coordonnées de $\mathscr{D}$, il est appelé seulement disque relatif à ces coordonnées. $\mathscr{D}$ est dit pseudoconvexe si la condition suivante est remplie. Pour une famille quelconque $\mathfrak{D}_{\iota}(\iota \in I)$ de disques analytiques dans $\mathscr{D}$, si $\cup \partial D_{\diamond}$ se trouve dans l'intérieur complet de $\mathscr{D}$, il en est ainsi pour $\cup D_{\iota}$. Du théorème de Levi, un domain de méromorphie sans point critique intérieur étalé au-dessus de $X$ est toujours pseudoconvexe.

Dénotons, en général, $l_{j}$ un ensemble analytique de la forme $a_{1} \times$ $\cdots \times a_{j-1} \times \boldsymbol{P}^{m_{j}} \times a_{j+1} \times \cdots \times a_{r}$ dans $X$, où $a_{i} \quad(i \neq j)$ sont des points quelconques de $\mathbb{P}^{m_{2}}$; et $L_{j}$ un ensemble compact et connexe dans $\mathscr{D}$ tel que l'on ait $\pi\left(L_{j}\right)=l_{j}$ pour un $l_{j}$. $L_{j}$ est, s'il existe, isomorphe à $l_{j}$ par $\pi$ puisque $l_{j}$ est simplement connexe, et que $\mathscr{D}$ n'a aucun point critique intérieur. On dénote, pour chaque $j, U_{j}$ l'ensemble de tous les points $a$ de $\mathscr{D}$ pour lesquel il existe un $L_{j}$ contenant $a$. Dans la suite on suppose que $\mathscr{D}$ soit pseudoconvexe sans rien en dire. On aura, d'abord, le

Lemme 1. Si, pour certain $j$, il existe au moins un $L_{j}, U_{j}$ coïncide avec tout $\mathscr{D}$.

En effet, $U_{j}$ est évidemment ouvert dans $\mathscr{D}$ puisque $L_{j}$ est tout compact dans $\mathscr{D}$. Scit $\left\{a^{(v)}\right\}(v=1,2, \ldots)$ une suite de points $a^{(v)}$ de $U_{j}$ qui tend vers un point $a^{(0)}$ de $\mathscr{D}$, et soient $L_{j}^{(v)}(v=1,2, \ldots)$ les ensembles analytique comme ci-dessus contenant $a^{(v)}$. Alors, la suite de $l_{j}^{(v)}=\pi\left(L_{j}^{(v)}\right)(v=1,2, \ldots)$ tend évidemment vers $l_{j}^{(0)}$ qui contient $\underline{a}^{(0)}$. Soit $\tilde{L}_{j}^{(0)}$ un composant connexe de $\pi^{-1}\left(l_{j}^{(0)}\right)$ qui contient $a^{(0)}$. Il est évidemment compact puisque $\mathscr{D}$ est pseudoconvexe. Cela signifie 
que $a^{(0)}$ se trouve dans $U_{j}$. Donc $U_{j}$ est fermé dans $\mathscr{D}$. Par suite, $U_{j}$ est ou vide ou bien identique à $\mathscr{D}$.

Il s'ensuit du lemme 1 quil existe au moins un de $j$, disons $s$, tel que $U_{s}$ soit vide pourvu que $\mathscr{D}$ ne soit pas identique à $X$; c'est-à-dire, pour tout point $a$ de $\mathscr{D}$, il n'y a aucun $L_{s}$ contenant $a$. Dénotons, en général, $\mathscr{D}_{s}(a)$ le composant connexe de $\pi^{-1}\left(l_{s}\right)$ qui contient $a$. On peut le regarder comme un domaine pseudoconvexe sans point critique intérieur étalé au-dessus de $\mathbb{P}^{m_{s}}$ qui n'est pas identique à $\mathbb{P}^{m_{s}}$.

3. Soit $\Lambda_{s}$ une métrique canonique de $p^{m n_{s}}$. Comme on le sait bein, l'élément linéaire $d s$ de $\Lambda_{s}$ est donné par

$$
d s^{2}=\frac{\sum\left|d z_{i}\right|^{2}}{1+\sum\left|z_{i}\right|^{2}}-\frac{\sum \bar{z}_{i} z_{j} d z_{i} d \bar{z}_{j}}{\left(1+\sum\left|z_{i}\right|^{2}\right)^{2}} \quad\left(i, j=1,2, \ldots, m_{s}\right)
$$

où $z_{i}\left(i=1, \ldots, m_{s}\right)$ sont les coordonnées inhomogènes convenables de $\mathbb{P}^{m_{s}}$. On prendra, dans toute la suite, des systèmes de coordonnées, dits admissibles pour $\Lambda_{s}$, tels que $\Lambda_{s}$ soit donné par la formule ci-dessus. Cela posé, on peut définir, pour chaque $\mathscr{D}_{s}(a)$, la distance frontière $d_{a}^{s}(p)$ mesurée par $\Lambda_{s}$. D'après M. Takeuchi, la fonction $-\log d_{s}^{a}(p)$ est plurisousharmonique sur $\mathscr{D}_{s}(a)$. Posons $d(p)=d_{s}^{a}(p)$ où $p$ est un point de $\mathscr{D}$ et $a$ est un point tel que $\mathscr{D}_{s}(a)$ contienne $p . d(p)$ est bien défini et est une fonction réelle positive sur $\mathscr{D}$. Alors, on aura le

Lemme 2. La fonction $-\log d(p)$ est aussi plurisous harmonique sur tout $\mathscr{D}$.

En effet, elle est évidemment supérieurement semicontinue puisque $\mathscr{D}$ est ouvert. Pour simplifier l'écriture, on suppose que $s=r$. Prenons un point quelconque $a$ de $\mathscr{D}$ et un système de coordonnées inhomogènes $z_{i}^{(j)}\left(i=1, \ldots, m_{j}, j=1, \ldots r\right)$ pour chaque $\mathbb{P}^{m_{j}}$ de façon que $\underline{a}$ est donné par $z_{j}^{(i)}=0\left(i=1, \ldots, m_{j}, j=1, \ldots, r\right) \quad$ et $z_{i}^{(r)}\left(i=1, \ldots, m_{r}\right)$ est admissible pour $\Lambda_{r}$. Soient $\Delta$ un disque quelconque dans $\mathscr{D}$ relatif à ces coordonnées dont le centre est $a$ et $u(p)$ une fonction harmonique sur $\Delta$ et continue sur sa fermeture $\bar{\Delta}$ telle que l'on ait

$$
-\log d(p) \leqq u(p)
$$


sur sa frontière $\partial \Delta$. Pour notre but actuel, il ne faut que montrer que l'on ait

$$
-\log d(a) \leqq u(a)
$$

Lorsque $\pi(\Delta)$ se trouve dans un $l_{r}$, cela a été démontré par M. Takeuchi. Donc on suppose qu'il n'en soit pas ainsi. Soit $b$ un point frontière de $\mathscr{D}_{r}(a)$ tel que $d(a)$ soit égal à la distance de $p_{r}(\underline{a})$ à $p_{r}(\underline{b})$ mesureé par $\Lambda_{r}$, et notons $N$ la droite complexe joignant $\underline{a}$ et $\underline{b}$. Maintenant on peut supposer sans restreindre la généralité que $N$ soit donné par $z_{i}^{(r)}=0\left(i=2, \ldots, m_{r}\right), z_{i}^{(j)}=0\left(j=1,2, \ldots, r-1, i=1, \ldots, m_{j}\right)$ et que $\pi(\Delta)$ se trouve dans la sous-variété linéaire $M \times N$ de $X$ où $M$ est donné par

$$
\begin{array}{ll}
z_{1}^{(i)}=\beta_{j} x, \quad z_{i}^{(j)}=0 & \left(i=2,,, m_{j}, j=1,,, r-1\right) \\
z_{2}^{(r)}=\beta_{r} x, \quad z_{i}^{(r)}=0 & \left(i=1,3, \ldots, m_{r}\right)
\end{array}
$$

où $x$ est une coordonnée de $M$. D'ailleurs on peut supposer que $\beta_{1}=1$, parce que, si tous les $\beta_{j}$ sont nuls, $\pi(\Delta)$ est contenu dans un $l_{r}$. En posant $z_{1}^{(1)}=y, \pi(\Delta)$ s'exprime par

$$
y=\alpha x \quad|x|<\rho,
$$

où $\alpha$ et $\rho$ sont une constante complexe et positive respectivement. Désignons $\mathscr{L}$ le composant connexe de $\pi^{-1}(M \times N)$ qui contient le point a. $\mathscr{L}$ peut se regarder comme un domaine pseudoconvexe sans point critique intérieur étalé au-dessus de $M \times N$ et on note $\pi_{1}$ la projection sur $M \times N$. D'après le lemme 1 , il n'y a aucune droite complexe dont l'image par $\pi_{1}$ est de la forme $x \times N$.

Donc on peut définir les deux distances frontières $d_{1}(p)$ et $e(p)$ mesurées par la restriction de $\Lambda_{r}$ sur $x \times N$ et la métrique projective respectivement dont $y$ est la coordonnée admissible sur $x \times N$. Notons $d(x), d_{1}(x)$ et $e(x)$ la restriction de $d(p), d_{1}(p)$ et $e(p)$ sur $\bar{\Delta}$ et regardons $d(x), d_{1}(x), e(x)$ et la fonction $u$ comme une fonction de $x$. D'après M. Takeuchi, deux point $x \times y_{1}$ et $x \times y_{2}$ étant donnés sur $x \times N$, les distances $d_{1 x}\left(y_{1}, y_{2}\right)$ et $e_{x}\left(y_{1}, y_{2}\right)$ mesurées par ces deux métriques respectivement sont données par 


$$
\begin{aligned}
e_{x}\left(y_{1}, y_{2}\right) & =\sin ^{-1} \frac{\left|y_{1}-y_{2}\right|}{\sqrt{1+\left|y_{1}\right|^{2}} \sqrt{1+\left|y_{2}\right|^{2}}}=\tan ^{-1} \frac{\left|y_{1}-y_{2}\right|}{\left|\bar{y}_{1} y_{2}+1\right|} \\
d_{1 x}\left(y_{1}, y_{2}\right) & =e_{x}\left(\frac{y_{1}}{\sqrt{1+\left|\beta_{r} x\right|^{2}}}, \frac{y_{2}}{\sqrt{1+\left|\beta_{r} x\right|^{2}}}\right) \\
& =\sin ^{-1} \frac{\left|y_{1}-y_{2}\right|}{\sqrt{1+\left|y_{1}\right|^{2}+\left|\beta_{r} x\right|^{2}} \sqrt{1+\left|y_{2}\right|^{2}+\left|\beta_{r} x\right|^{2}}}
\end{aligned}
$$

Considérons un domaine dans $\{|x| \leqq \rho\} \cap M \times N$

$$
D_{c}=\left\{(x, y) ; e_{x}(\alpha x, y)-u(x)+c<0\right\}
$$

où $c$ est une constante réelle. Comme $\bigcap_{n=1}^{\infty} D_{n}=\pi_{1}(\Delta), D_{c}$ peut se regarder comme un domaine de $\mathscr{L} \cap\{|x| \leqq \rho\}$ pour tout $c$ suffisamment grand. Soit $c_{0}$ le nombre le plus petit tel que $D_{c}$ puisse être regardé comme un domaine dans $\mathscr{L} \cap\{|x| \leqq \rho\}$ pour tout $c$ plus grand que $c_{0}$. Alors il existe au moins un point frontière $p_{0}$ commun de $\mathscr{L}$ et de $D_{c_{0}}$ dont on note $\left(x_{0}, y_{0}\right)$ les coordonnées. Si $c_{0}>0$, on aurait nécessairement $\left|x_{0}\right|<\rho$ parce que $e(x) \geqq d_{1}(x) \geqq d(x)$ vu leurs définitions et $-\log d(x) \leqq$ $u(x)$ sur $\partial \Delta$. Or $e_{x}(\alpha x, y)-u(x)$ est fortement plurisurharmonique dans $\{|x| \leqq \rho\} \cap M \times N-\pi(\Delta)$, que l'on voit par calculer explicitement la matrice hessienne complexe. En conséquence, on pourrait construire facilement une famille $\left\{\boldsymbol{D}_{\iota}\right\}_{\iota \in J}$ de disques analytiques dans $\mathscr{L}$ telle que $U \partial \boldsymbol{D}_{\imath}$ se trouve dans l'intérieur complet de $\mathscr{L}$ mais $\overline{\cup D}$ contienne $p_{0}$. Donc il faut que $c_{0} \leqq 0$ et par suite $-\log d(0) \leqq u(0)$ vu que $d(0)=e(0)$. Cela signifie que la fonction $-\log d(p)$ est plurisousharmonique. Ce lemme a été donc démontré certainement.

4. Soit, de nouveau, $\mathscr{D}$ un domaine pseudoconvexe sans point critique intérieur étalé au-dessus de $X=\boldsymbol{P}^{m_{1}} \times \cdots \times \boldsymbol{P}^{m_{r}}$. On suppose que $\mathscr{D}$ ne coïncide pas avec $X$. Par suite, il existe au moins un de $U_{j}(j=1, \ldots, r)$, disons $U_{s}$, qui est vide. Maintenant, on suppose, pour réduire à l'absurde, que $\mathscr{D}$ contienne un ensemble analytique compact $A$ tel que la projection $p_{s}(\pi(A))$ de $A$ dans $\mathbb{P}^{m_{s}}$ soit de dimension positive. Evidemment, la fonction $d(p)$ définie dans la section précédente est égale à une constante $\alpha$ sur $A$, puisque la restriction de $-\log d(p)$ sur $A$ est aussi plurisousharmonique. Prenons deux points $a$ et $b$ sur 
$A$ de manière que l'on ait $p_{s}(\underline{a}) \neq p_{s}(\underline{b})$. Alors, pour un voisinage $\delta$ de $b$, quelque petit qu'il soit, on peut toujours trouver un point $b^{\prime}$ de $\delta$ tel que l'on ait $d(b)>d\left(b^{\prime}\right)$. Soit $T_{b^{\prime}}^{s}$, une transformation automorphe de $\boldsymbol{P}^{m_{s}}$ qui fait correspondre à $p_{s}(\underline{a})$ lui-même et à $p_{s}(\underline{b})$ le point $p_{s}\left(b^{\prime}\right)$ et définissons une transformation automorphe $T_{b^{\prime}}$ de $X$ par

$$
T_{b^{\prime}}=I_{1} \times \cdots \times I_{s-1} \times T_{b^{\prime}}^{s} \times I_{s+1} \times \cdots \times I_{r}
$$

où $I_{j}$ signifie la transformation identique de $\boldsymbol{P}^{m_{j}}(j \neq s)$. Alors, on peut trouver un autre ensemble analytique irréductible compact $A^{\prime}$ contenant $a$ et $b^{\prime}$ dans $\mathscr{D}$ tel que l'on ait $\pi\left(A^{\prime}\right)=T_{b^{\prime}}(\underline{A})$, pourvu que $b^{\prime}$ se trouve suffisamment voisin de $b$. De la même raison comme ci-dessus, $d(p)$ est égal à une constante $\alpha^{\prime}$ sur $A^{\prime} . A$ et $A^{\prime}$ ayant un point commun $a, \alpha$ doit être égal à $\alpha^{\prime}$. C'est évidemment absurde, et, par suite, s'il existe un tel ensemble analytique $A$, $\mathscr{D}$ est identique à $U_{s}$ vu le lemme 1. D'après le lemme 1 at ce que l'on vient de dire, on a le.

Théorème 1. Un domaine pseudoconvexe sans point critique intérieur étalé au-dessus de $X$, s'il ne coïncide pas avec $X$, ne contient aucun ensemble analytique compact $A$ tel que, pour chaque $j(j=1, \ldots$, $r), p_{j}(\pi(A))$ soit de dimension positive.

$\mathrm{Du}$ théorèm 1 , on aura facilement le

Théorème 2. Soit $A$ un ensemble analytique compact dans $X$ tel que, pour chaque $j(j=1, \ldots, r), p_{j}(A)$ soit de dimension positive. Alors, toute fonction méromorphe $f$ dans un voisinage $\mathfrak{B}$ de $A$ est la restriction à $\mathfrak{B}$ d'une fonction méromorphe sur tout $X$.

En effect, par le prolongement analytique de $f$ à partir de $\mathfrak{B}$ sur $X$, on a un domaine de méromorphie $\mathscr{D}$ de $f$ sans point critique intérieur étalé au-dessus de $X$. En ce moment, du théorème $1, \mathscr{D}$ ne peut avoir aucun point frontière puisque $\mathscr{D}$ contient l'ensemble analytique $A$. De plus, puisque $X$ est simplement connexe, $\mathscr{D}$ doit être identique à $X$. Donc, le théorème a été démontré certainement. 


\section{Bibliographie}

[1] Barth, W., Fortsetzung meromorpher Funktionen in Tori und komplexer-projectiven Räumen, Invent Math. 5 (1968), 42-62.

[2] Chow, W. L., On meromorphic maps of algebraic varieties, Ann. of Math. (2) 89, (1969), 391-403.

[3] Fujita, R., Domaines sans point critique intérieur sur l'espace projectif complexe, J. Math. Soc. Japan 15 (1963), 443-473.

[4] Hironaka, H. and Matsumura, H., On meromorphic maps of algebraic varieties, J. Math. Soc. Japan 20 (1968), 52-82.

[5] Rossi, H., Continuation of subvarieties of projective varieties, Amer. J. Math. 91 (1969), 565-575.

[6] Takeuchi, A., Domaines pseudoconvexes infinis et la métrique riemannienne dans un espace projectif, J. Math. Soc. Japan 16 (1964), 159-181. 
